\title{
RNAi screening of the human colorectal cancer genome identifies multifunctional tumor suppressors regulating epithelial cell invasion
}

Cell Research (2012) 22:1605-1608. doi:10.1038/cr.2012.140; published online 9 October 2012

\section{Dear Editor,}

Genomic sequencing of human tumor-derived DNA has identified a vast number of novel tissue-specific genetic mutations occurring at varying frequencies [1]. Frequently mutated genes are informally referred to as driver alterations (or candidate cancer genes, $C A N$ genes), whereas those less frequently mutated are considered passenger alterations $[1,2]$. With few exceptions, the majority of mutations are loss-of-function alterations and the ones that occur rarely are presumed to be a byproduct of genomic instability or normal mutation rates that do not directly contribute to tumor progression (passengers) [3]. In addition to computational and biostatistical models for parsing driver from passenger mutations, the utility of biological assays to functionally interrogate cancer genomes is required to determine which of these mutations are able to contribute to a particular tumorigenic phenotype (i.e., anchorage-independent growth, resistance to apoptosis, enhanced invasion through extracellular matrices). Moreover, these assays can also decipher whether less frequently mutated genes are actively involved in cancer progression. Thus, the development of experimental methods to separate putative driver from passenger mutations is critical towards understanding which genes are directly promoting tumorigenesis. Although sequencing of the human colorectal cancer (CRC) genome, in parallel with computational analyses, has detected 140 frequently mutated $C A N$-genes and up to $\sim 700$ mutations occurring at a lower frequency (passengers) [1], it is difficult to distinguish whether these mutations play a causal or incidental role in tumor development. The identification of those mutated genes with tumorassociated roles may therefore lead to novel therapeutic avenues as we transition towards an era of personalized medicine and targeted therapy.

Advances in RNA interference (RNAi) technologies have enabled loss-of-function screens to identify tumor suppressors in mammalian cells, the knockdown of which promotes an assayable cancer-associated phenotype(s) [4]. Compared to whole-genome screens, RNAi screens focusing on specific gene sets obtained from cancer genome sequencing data are suitable for classifying potential driver from passenger alterations. To identify tumor suppressors of human CRC, we conducted a loss-of-function screen using a small interfering RNA (siRNA) library (Dharmacon, Lafayette, CO) targeting the most frequently mutated genes in CRC (CANgenes) for the ability to permit cell invasion, a hallmark of cancer. The initial step in the progression of a localized tumor towards invasive and metastatic disease occurs when cells acquire the ability to invade through a basement membrane [5]. In this screen, we utilized a modified Boyden chamber coated with Matrigel (BD Biosciences, San Jose, CA), a reconstituted extracellular matrix, to recapitulate these events [6] using normal migratory, yet non-invasive, epithelial cells. These screens were carried out with non-malignant, Cdk4- and hTERTimmortalized human colonic epithelial cells (HCEC) [7] in the background of either TP53-knockdown (1CTP) using short hairpin RNAs (shRNA) or ectopic expression of oncogenic $K R A S^{V 12}$ (1CTR) [8]. The gene sets used in this study (CAN-genes) were derived from the study of Wood et al [1]. According to this study, $A P C, K R A S$, TP53, and PIK3CA are mutated most often across a panel of sequenced colon tumors.

Figure 1A represents a diagram of the screening scheme. Briefly, pooled siRNAs (four siRNAs per gene, sequences listed in Supplementary information, Table S1, methods listed in Supplementary information, Data S1) were reverse-transfected into 1CTP and 1CTR HCECs in $2 \%$ serum growth medium for $24 \mathrm{~h}$ before cells were washed and serum-starved overnight. Cells transfected for $48 \mathrm{~h}$ were then harvested in serum-free medium and plated onto 24-well Matrigel-coated transwell filters (BD Biosciences, San Jose, CA). The bottom chamber 
A

\begin{tabular}{cccc} 
Day 1 & Day 2 & Day 3 & Day 4 \\
\hline $\begin{array}{c}\text { Reverse transfection of } \\
\text { pooled siRNAs }\end{array}$ & $\begin{array}{c}\text { Wash and serum- } \\
\text { starve overnight }\end{array}$ & $\begin{array}{c}\text { Invasion assay over } \\
\text { Matrigel transwell }\end{array}$ & $\begin{array}{c}\text { Fix and stain } \\
\text { invaded cells }\end{array}$
\end{tabular}

B

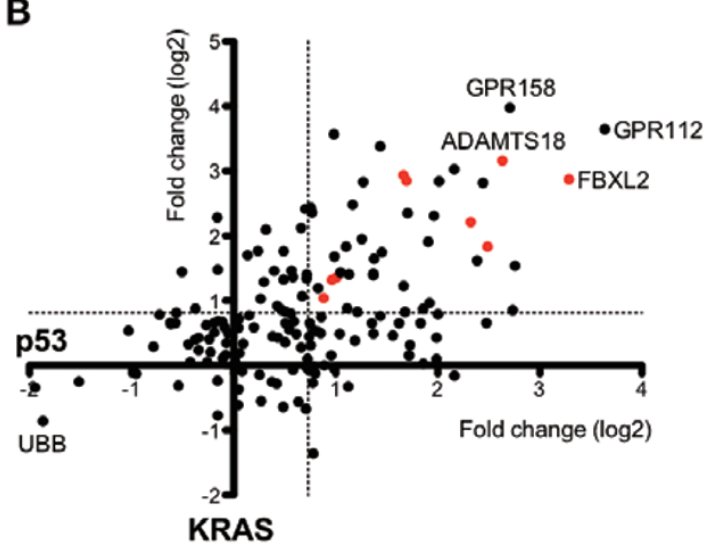

C

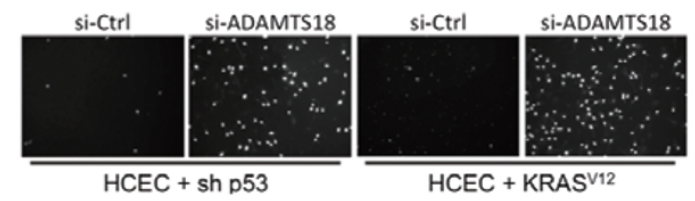

D

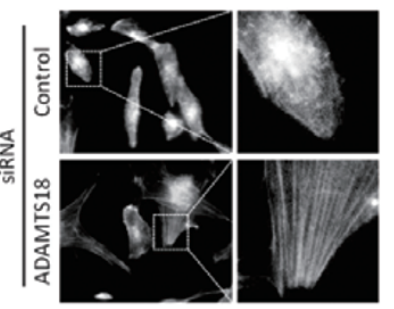

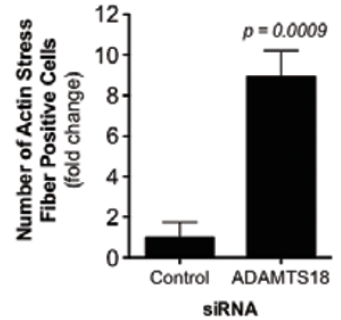

$\mathbf{F}$

$F$
E

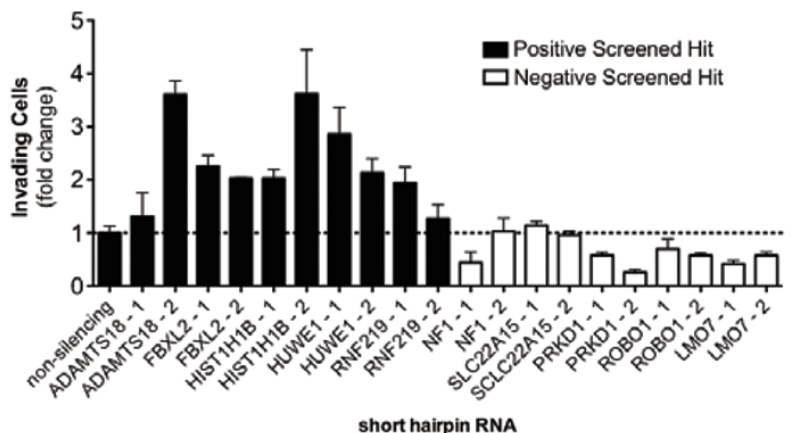

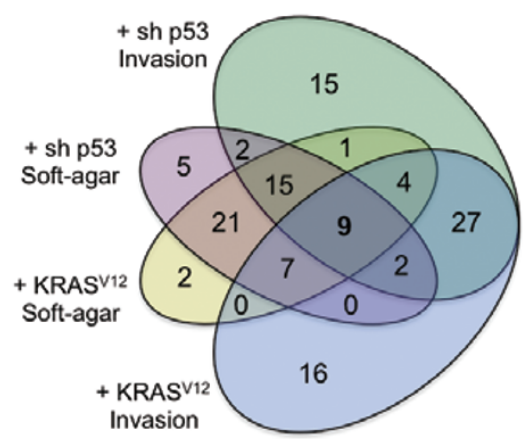

G

Context-independent multifunctional tumor suppressors

\begin{tabular}{|c|c|c|c|c|}
\hline \multicolumn{5}{|c|}{ Invasion (fold change \pm SD) } \\
\hline Gene & Gene Name & Entrez Gene ID & TP53 & KRAS \\
\hline ADAMTS18 & A disintegrin and metalloproteinase with thrombospondin motif 18 & 170692 & $13.9 \pm 2.6$ & $23.6 \pm 2.7$ \\
\hline$F B X L 2$ & F-box and leucine-rich repeat protein 2 & 25827 & $26.7 \pm 6.3$ & $17.7 \pm 5.3$ \\
\hline HIST1H1B & Histone cluster $1, \mathrm{H} 1 \mathrm{~b}$ & 3009 & $5.3 \pm .04$ & $18.9 \pm 4.9$ \\
\hline RNF219 & Ring finger protein 219 & 72486 & $5.4 \pm 1.8$ & $17.3 \pm 2.3$ \\
\hline HUWE1 & HECT, UBA, and WWE domain containing 1, E3 ubiquitin ligase & 10075 & $12.0 \pm 2.8$ & $6.3 \pm 2.5$ \\
\hline MAP2K7 & Mitogen-activated protein kinase kinase 7 & 5609 & $10.2 \pm 2.9$ & $9.1 \pm 5.1$ \\
\hline PTPRD & Protein tyrosine phosphatase, receptor type, D & 5789 & $2.8 \pm 0.2$ & $3.9 \pm 1.5$ \\
\hline TAF2 & RNA polymerase II, TATA box binding protein-associated factor & 170844 & $2.6 \pm 0.3$ & $3.7 \pm 1.3$ \\
\hline TGFBR2 & Transforming growth factor, beta receptor 2 & 7048 & $2.4 \pm 0.5$ & $2.8 \pm 1.2$ \\
\hline
\end{tabular}

Figure 1 (A) Overall scheme of focused siRNA screen for tumor suppressors involved in regulating the invasive potential of normal cells. 1CTP (shRNA-TP53) and 1CTR (oncogenic-KRAS ${ }^{V 12}$ ) HCECs were reverse-transfected $48 \mathrm{~h}$ with pooled siRNAs prior to re-plating onto Matrigel-coated transwell filters. Invaded cells were Hoechst-stained and data were normalized to non-targeting siRNA control. (B) Scatterplot of the overall screening results. The x-axis represents fold changes in invasion in 1CTP cells, whereas the $y$-axis represents those for 1CTR cells. Each dot represents a single gene targeted by pooled siRNAs (four siRNAs/gene) and red dots represent multifunctional tumor suppressor genes (listed in G). The dotted line represents the statistical cutoff point and is defined as three standard deviations. (C) Representative images of Hoechststained invaded 1CTP and 1CTR cells with control siRNA or siRNA targeting the ADAMTS18 gene. (D) Depletion of ADAMTS18 using siRNAs induces the formation of F-actin stress fibers. (E) Screening results were partially validated in 1CTP cells using shRNA-mediated knockdown of five positive hits and five negative non-hits. Fold change in invasion is normalized to non-silencing shRNA. (F) Venn diagram of overlapping soft-agar growth and invasion suppressing genes in each cell background. Soft-agar hits were obtained from a previously published screen [8]. Nine genes were identified through comparative analysis of both screens. RNAi knockdown of these genes leads to multiple tumorigenic phenotypes. (G) List of the nine context-independent multifunctional tumor suppressor hits. Knockdown of these nine genes enhances both anchorage-independent growth and invasion in two independent backgrounds in vitro (also shown as red dots in B). Bar graphs are depicted as mean \pm SEM. 
is filled with $2 \%$ serum medium plus growth factors as a chemoattractant. After overnight incubation, invaded cells were then fixed for Hoechst staining (Invitrogen, Grand Island, NY). Images were taken at $10 \times$ with five fields imaged per well. Altogether, pooled siRNAs targeting 159 genes (140 CAN-genes plus 19 additional "passenger" genes that closely interacted with $C A N$-genes through interaction mapping [8]) were tested in two cell lines (1CTP and 1CTR) for the ability to enhance invasion in vitro. The overall screening results are shown in Figure $1 \mathrm{~B}$ as a scatterplot wherein the $\mathrm{x}$ - and $\mathrm{y}$-axis represent the fold changes in invasion in the background of 1CTP and 1CTR cells, respectively. siRNAs targeting the essential ubiquitin B (UBB) gene were used to determine transfection efficiency and as a negative control, and a non-targeting control siRNA was used for normalization. A statistical cutoff point of three standard deviations was used to eliminate noise and false positives.

Depletion of 42 out of 159 (26.4\%) screened genes promoted invasion in both cell backgrounds in a contextindependent manner. The overall distribution of all siRNA pools screened in both backgrounds (calculated as changes in cell invasion) is depicted graphically in Supplementary information, Figure S1 and numerically in Supplementary information, Tables S2 and S3. The results of the twenty most potent suppressors of invasion in each context are shown in Supplementary information, Figure S2. Among these, knockdown of two G-protein coupled receptors (GPR112 and GPR158) with previously unidentified roles in cancer progression was among the most effective in inducing cell invasion. Moreover, Figure 1C shows representative screening images of Hoechst-stained invaded cells on a transwell filter where depletion of ADAMTS18 levels by pooled siRNAs induces context-independent cell invasion. Deconvolution of pooled ADAMTS18 siRNAs revealed that three out of four siRNAs tested positive for increased cell invasion, strongly suggesting "on-target" effects of RNAi (Supplementary information, Figure S3). Since stress fiber formation has been associated with cell migration and focal adhesion assembly that may promote cell invasion [9], as a proof of principle experiment, we next determined whether ADAMTS18 depletion could lead to rearrangements in the actin cytoskeleton. siRNA-mediated knockdown of ADAMTS18 in 1CTP HCECs induced an approximate nine-fold increase in the number of cells positive for actin stress fiber formation compared to a non-targeting control siRNA (Figure 1D).

Next, we sought to validate several of the identified hits using stable shRNA knockdowns (pGIPZ library, Open Biosystems, Lafayette, $\mathrm{CO}$ ). We chose to retest five genes among each group that scored positive in the
siRNA screen and five random genes that scored negative using two shRNAs per gene in 1CTP cells. A nontargeting shRNA was used for normalization. On average, shRNAs targeting genes that scored positive from the screen (black bars) resulted in a $2.3 \pm 0.8$-fold change in invasion compared to $0.6 \pm 0.3$-fold change using shRNAs against negatively scored genes (white bars) (Figure $1 \mathrm{E})$. Quantitative real-time PCR were performed on four of the positive hits (FBXL2, HIST1H1B, HUWE1, and $R N F 219)$ to determine shRNA knockdown efficiency (primer sequences listed in Supplementary information, Table S4). Seven out of eight hairpins caused marked reduction in target gene mRNA levels (Supplementary information, Figure S4), with the exception of one shRNA targeting HISTIH1B.

We have previously completed similar loss-of-function screens for tumor suppressors, the knockdown of which allows for bypass of anchorage-dependent proliferation restraints (i.e., growth in soft-agar) [8]. From those studies, we identified 52 context-independent regulators of soft-agar growth representing $\sim 33 \%$ of all $C A N$-genes, an unexpectedly high percentage. Cross-analysis with this previously completed screen for soft-agar growth revealed that nine out of the 42 invasion-suppressing genes identified in the current study also permitted anchorageindependent growth upon knockdown (Figure $1 \mathrm{~F}$ and Supplementary information, Table S5). These nine overlapping genes represent those mutated at a high frequency in human CRC with multiple tumorigenic phenotypes when depleted in vitro in a context-independent manner (Figure 1G). Furthermore, the effect of these siRNAs on HCEC proliferation is shown in Supplementary information, Figure S5.

One of the most potent suppressors of invasion that also conferred soft-agar growth upon RNAi-mediated knockdown is the ADAMTS18 gene. ADAMTS18 (a disintegrin and metalloproteinase with thrombospondin motifs $\underline{18}$ ) belongs to a family of ADAMTS peptidases with well-studied roles in extracellular matrix degradation and reorganization. Mutations in ADAMTS18 have previously been linked to melanoma progression [10]. ADAMTS18 is mutated in $6 \%$ of colorectal carcinomas out of 193 sequenced tumors (The Cancer Genome Atlas), representing a large number of patients out of the $\sim 140000$ annually diagnosed colon cancer cases in the United States alone (American Cancer Society).

In summary, these results allow for deconstructing driver and passenger alterations within a given tumor context characterized by cancer-associated phenotypes. Loss-of-function screening using RNAi provides a robust method to rapidly analyze the cellular effects of depleting specific genes identified from cancer genome sequenc- 
ing efforts. While not comprehensive of all the genomic changes that occur in CRC, these studies are an initial step in validating and complementing bioinformatic approaches in selecting the most critical genes to pursue for future therapeutic discovery. The other multifunctional tumor suppressors identified from comparative analysis of the two independent screens include a group of ubiquitin ligases (FBXL2, RNF219, HUWE1), a histone protein $(H I S T 1 H 1 B)$, cell signaling components (MAP $2 K 7$, $P T P R D, T G F B R 2$ ), and a subunit of transcription factor II D (TAF2). Future studies will involve screening candidate driver mutations for the ability to bypass CRC chemotherapy-induced apoptosis (i.e., mutations promoting resistance to 5 -fluorouracil treatment).

\section{Acknowledgments}

This work is supported by CPRIT Training Grant (RP101496) to PL, NASA Grants (NNX09AU95G, NNX11AC15G and NNX$11 \mathrm{AC} 54 \mathrm{G})$ to JWS, and NCI SPORE (CA70907) to JWS.

Peter Ly ${ }^{1}$, Ugur Eskiocak ${ }^{1}$, Chelsea R Parker ${ }^{1}$, Kenneth J Harris ${ }^{1}$, Woodring E Wright ${ }^{1}$, Jerry W Shay ${ }^{1,2}$

${ }^{1}$ Department of Cell Biology, University of Texas Southwestern Medical Center, Dallas, TX 75390, USA; ${ }^{2}$ Center of Excellence in Genomic Medicine Research, King Abdulaziz University, Jeddah, Saudi Arabia

Correspondence: Jerry W Shay

Tel: 214-648-3282

E-mail: jerry.shay@utsouthwestern.edu

\section{References}

1 Wood LD, Parsons DW, Jones S, et al. The genomic landscapes of human breast and colorectal cancers. Science 2007; 318:1108-1113.

2 Stratton MR. Exploring the genomes of cancer cells: progress and promise. Science 2011; 331:1553-1558.

3 Carter H, Chen S, Isik L, et al. Cancer-specific highthroughput annotation of somatic mutations: computational prediction of driver missense mutations. Cancer Res 2009; 69:6660-6667.

4 Schlabach MR, Luo J, Solimini NL, et al. Cancer proliferation gene discovery through functional genomics. Science 2008; 319:620-624.

5 Sethi N, Kang Y. Unravelling the complexity of metastasismolecular understanding and targeted therapies. Nat Rev Cancer 2011; 11:735-748.

6 Albini A, Iwamoto Y, Kleinman HK, et al. A rapid in vitro assay for quantitating the invasive potential of tumor cells Cancer Res 1987; 47:3239-3245.

7 Roig AI, Eskiocak U, Hight SK, et al. Immortalized epithelial cells derived from human colon biopsies express stem cell markers and differentiate in vitro. Gastroenterology 2010; 138:1012-1021.

8 Eskiocak U, Kim SB, Ly P, et al. Functional parsing of driver mutations in the colorectal cancer genome reveals numerous suppressors of anchorage-independent growth. Cancer Res 2011; 71:4359-4365

9 Chrzanowska-Wodnicka M, Burridge K. Rho-stimulated contractility drives the formation of stress fibers and focal adhesions. J Cell Biol 1996; 133:1403-1415.

10 Wei X, Prickett TD, Viloria CG, et al. Mutational and functional analysis reveals ADAMTS18 metalloproteinase as a novel driver in melanoma. Mol Cancer Res 2010; 8:1513-1525.

(Supplementary information is linked to the online version of the paper on the Cell Research website.) 